\title{
NONANALYTIC SOLUTIONS OF CERTAIN LINEAR PDE'S
}

BY

\author{
E. C. ZACHMANOGLOU
}

\begin{abstract}
It is shown that if $P$ is a linear partial differential operator with analytic coefficients, and if $M$ is an analytic submanifold of codimensions 3 in $\mathbf{R}^{n}$, which is partially characteristic with respect to $P$ and satisfies certain additional conditions, then one can find, in a neighborhood of any point of $M$, solutions of the equation $P u=0$ which are flat or singular precisely on $M$. The additional condition requires that a nonhomogeneous Laplace equation in two variables possesses a solution with a strong extremum at the origin. The right side of this nonhomogeneous equation is a homogeneous polynomial in two variables with coefficients being repeated Poisson brackets of the real and imaginary parts of the principal symbol of $P$.
\end{abstract}

1. Introduction. Let $P=P(x, D)$ be a linear partial differential operator of order $m \geqslant 1$ with complex valued coefficients which are defined and analytic in an open subset $\Omega$ of $\mathbf{R}^{n}$. Theorem 2 of [1] asserts that if $M$ is an analytic manifold in $\Omega$, which is partially characteristic with respect to $P$ and satisfies certain additional conditions, then one can find, in a neighborhood of any point of $M$, solutions of the homogeneous equation

$$
P(x, D) u=0
$$

which are (a) flat on $M$ and analytic and nowhere vanishing in its complement, or (b) singular on $M$ and analytic in its complement. In [3] it is shown that, under the same assumptions on $M$, one can find solutions of (1.1) which are flat or singular precisely on any analytic submanifold of $M$. In [4] new assumptions were introduced under which one can again find solutions of (1.1) which are flat or singular precisely on $M$. A basic new feature of [4] is that at a point $(x, \xi)$ where the principal symbol $p_{m}(x, \xi)$ of $P$ vanishes, it is assumed that the $\xi$-gradients of the real and imaginary parts of $p_{m}$ are linearly independent.

In this paper we go much further in the direction of [4] and express the condition which guarantees the existence of solutions of (1.1) which are flat or singular on $M$, by requiring that a certain nonhomogeneous two-dimensional Laplace equation has a solution possessing a strong extremum.

Let us recall some notation. We set

$$
A(x, \xi)=\operatorname{Re} p_{m}(x, \xi), \quad B(x, \xi)=\operatorname{Im} p_{m}(x, \xi)
$$

and denote by $H_{A}$ and $H_{B}$ the Hamilton fields of $A$ and $B$,

$$
H_{A}=\sum_{j=1}^{n}\left(\frac{\partial A}{\partial \xi_{j}} \frac{\partial}{\partial x_{j}}-\frac{\partial A}{\partial x_{j}} \frac{\partial}{\partial \xi_{j}}\right)
$$

Received by the editors June 16, 1982.

1980 Mathematics Subject Classification. Primary 35A05, 35F05, 35G05, 35H05; Secondary 35J05. 
$H_{A} B$ is known as the Poisson bracket of $A$ and $B$. For any point $\gamma=(x, \xi)$ in the cotangent bundle $T^{*} \Omega$, Hörmander's integer $k$ [2] is defined by

$$
\begin{aligned}
k=k(\gamma ; A, B)=\sup \left\{j: j \in \mathbf{N}, H_{C_{1}} \cdots H_{C_{l-1}} C_{l}(\gamma)=0,\right. & \\
& \text { for } \left.1 \leqslant l \leqslant j \text { and } C_{i}=A \text { or } B, i \geqslant 1\right\} .
\end{aligned}
$$

Thus $k$ is the smallest integer such that there is at least one repeated Poisson bracket of $A$ and $B$ with $k H$ 's which does not vanish at $\gamma$.

The theorem proved in this paper requires that the manifold $M$ is of codimension 3. For simplicity in this introduction we state the result for the special case in which $M$ is a point in $\mathbf{R}^{3}$.

"Let $x^{0} \in \Omega \subset \mathbf{R}^{3}$ and suppose that for some $\xi^{0} \in \mathbf{R}^{3} \backslash 0$ :

(i) $p_{m}\left(x^{0}, \xi^{0}\right)=0$,

(ii) $\operatorname{grad}_{\xi} A\left(x^{0}, \xi^{0}\right)$ and $\operatorname{grad}_{\xi} B\left(x^{0}, \xi^{0}\right)$ are linearly independent,

(iii) in a neighborhood of the origin of the $(t, s)$-plane, there is a homogeneous solution $v=v(t, s)$ of the equation

$$
v_{t t}+v_{s s}=\left.\left(t H_{A}+s H_{B}\right)^{k-1} H_{A} B\right|_{\left(x^{0}, \xi^{0}\right)}
$$

having a strong extremum at the origin. Then, in a neighborhood of $x^{0}$ one can find solutions of (1.1) which are flat or singular precisely at $x^{0}$."

It should be noted that the right side in (1.5) is a homogeneous polynomial of degree $k-1$ in $t$ and $s$, where $k=k\left(x^{0}, \xi^{0} ; A, B\right)$ is defined by (1.4). Thus the homogeneous solution $v$ of equation (1.5) required by condition (iii) is a homogeneous polynomial of degree $k+1$ in $t$ and $s$. Moreover condition (iii) implies that $k$ must necessarily be odd. Obviously, when $k=1$, condition (iii) is always satisfied since the right side of (1.5) is, in this case, the nonzero constant $\left.H_{A} B\right|_{\left(x^{0}, \xi^{0}\right)}$.

When $k=3$, equation (1.5) is

$$
v_{t t}+v_{s s}=a t^{2}+2 b t s+c s^{2}
$$

where

$$
\begin{gathered}
a=\left.H_{A}^{2} H_{A} B\right|_{\left(x^{0}, \xi^{0}\right)}, \quad b=\left.H_{A} H_{B} H_{A} B\right|_{\left(x^{0}, \xi^{0}\right)}=\left.H_{B} H_{A} H_{A} B\right|_{\left(x^{0}, \xi^{0}\right)}, \\
c=\left.H_{B}^{2} H_{A} B\right|_{\left(x^{0}, \xi^{0}\right)} .
\end{gathered}
$$

It can be shown that the necessary and sufficient condition for equation (1.6) to have a homogeneous solution with a strong extremum at the origin is

$$
(a+c)^{2}+32\left(a c-b^{2}\right)>0 .
$$

Thus, in the case $k=3$, assumption (iii) can be replaced by the more explicit condition (1.8) involving the repeated Poisson brackets of $A$ and $B$ with $3 H$ 's. This condition is weaker than the one used in [4], namely $a c-b^{2}>0$, and does not require that the right side of (1.6) is a positive definite quadratic form. Indeed when $a=1$ and $b=0,(1.8)$ is satisfied if and only if $c>-17+\sqrt{(17)^{2}-1}$.

An illustration is provided by the vector field in $\mathbf{R}^{3}$,

$$
L=\partial_{t}+i \partial_{s}+i g(t, s) \partial_{x},
$$


where $g(0, s) \equiv 0$. Assumptions (i) and (ii) are obviously satisfied if $x^{0}$ is given by $(x, t, s)=(0,0,0)$ and $\xi^{0}$ by $(\xi, \tau, \sigma)=(1,0,0)$. Moreover $H_{A}=\partial_{t}$, and since $H_{A} B=g_{t} \xi$ depends only on $t, s$ and $\xi$, we may take $H_{B}=\partial_{s}$ in the computation of the repeated Poisson brackets of $A$ and $B$. Thus the integer $k$ at $\left(x^{0}, \xi^{0}\right)$ is the order of the lowest order terms in the Taylor expansion of $g$ and the right side of (1.5) if $(k-1)$ ! times the $(k-1)$-order terms of $g_{t}$.

The statement of our theorem for a manifold of codimension 3 in $\mathbf{R}^{n}$ is given in $\S 2$. As in [1] the proof is based on the construction of a complex phase function, i.e. a solution of the characteristic equation

$$
p_{m}\left(x, \phi_{x}\right)=0
$$

in some open neighborhood $U$ of a point $x^{0}$ on the manifold $M$, possessing the following properties: (a) $\phi(x)=0$ if and only if $x \in M \cap U$, (b) the values of $\phi$ in $C$ avoid the negative imaginary half-axis and (c) $\operatorname{grad} \phi \neq 0$. Once such a phase function has been constructed the end of the proof of the theorem proceeds as in $\S 6$ of [1].

In $\S 3$ we show that the assumptions of our theorem are invariant under multiplication of $p_{m}$ by a nonvanishing function. Indeed equation (1.5) itself remains invariant under such a multiplication. The invariance is needed in the reduction of the principal symbol to a first order symbol. This reduction is contained in $\$ 4$ where we also express condition (iii) in terms of appropriate local coordinates. The desired phase function is constructed in $\$ 5$ by solving a Cauchy problem with data containing $a(k+1)$-order term with complex coefficient, the real and imaginary parts of which are chosen to be certain multiples of two of the coefficients of a solution of (1.5) having a strong extremum at the origin.

\section{Statement of results.}

THEOREM 2.1. Let $M$ be an analytic manifold in $\Omega$ with $\operatorname{codim} M=3$ and suppose that there is an analytic hypersurface $S$ containing $M$ such that for every $\gamma=(x, \xi) \in \Sigma$, where

$$
\Sigma=\left.N^{*}(S)\right|_{M}=\{(x, \xi) ; x \in M, \xi \neq 0 \text { normal to } S \text { at } x\},
$$

the following conditions are satisfied:

(I) $p_{m}(\gamma)=0$

(II) The dimension of the space spanned by $T_{x} M$ and by the base projections of $H_{A}(\gamma)$ and $H_{B}(\gamma)$ is $n-1$.

(III) $k=k(\gamma ; A, B)$ is constant.

(IV) In a neighborhood of the origin of the $(t, s)$-plane, there is a homogeneous solution $v=v(t, s)$ of the equation

$$
v_{t t}+v_{s s}=\left.\left(t H_{A}+s H_{B}\right)^{k-1} H_{A} B\right|_{\gamma}
$$

having a strong extremum at the origin. Then, if $x^{0}$ is any point of $M$, the following conclusions hold:

(a) There is an open neighborhood $U$ of $x^{0}$ in $\Omega$ and a solution $u$ of (1.1) in $U$ such that $u \in C^{\infty}(U), u$ is flat on $M \cap U$ and $u$ is analytic and nonvanishing in $U \backslash M$. 
(b) For any integer $p \geqslant m$ there is an open neighborhood $U_{p}$ of $x^{0}$ in $\Omega$ and a solution $u$ of $(1.1)$ in $U_{p}$ such that $u \in C^{p}\left(U_{p}\right)$, $u$ is analytic in $U_{p} \backslash M$ and $u$ is not $C^{p+1}$ in any neighborhood of any point of $M \cap U_{p}$.

REMARK. Condition (IV) implies that the constant value of $k$ on $\Sigma$ must be odd. Moreover, (IV) is always satisfied when $k=1$.

3. Invariance of assumptions. The assumptions of Theorem 2.1 are obviously invariant under analytic transformations of coordinates. This invariance is needed in the reduction of $p_{m}$ to a first order symbol. For this reduction it is also necessary to show that the assumptions are invariant under multiplication of $p_{m}$ by a nonvanishing function. For assumptions (II) and (III) this was done in [4]. For assumption (IV) we have

LEMMA 3.1. Equation (2.2) (and hence assumption (IV)) is invariant under multiplication of $p_{m}$ by a nonvanishing function.

Proof. Let

$$
q=\alpha+i \beta, \quad \alpha^{2}+\beta^{2} \neq 0,
$$

be a nonvanishing function defined and analytic near the fixed point $\gamma^{0} \in \Sigma$ and set

$$
\tilde{p}_{m}=q p_{m}=(\alpha+i \beta)(A+i B)=\tilde{A}+i \tilde{B}
$$

where $\tilde{A}=\alpha A-\beta B, \tilde{B}=\beta A+\alpha B$. Introducing now the real parameters $t$ and $s$ we find that

$$
t H_{\tilde{A}}+s H_{\tilde{B}}=t^{\prime} H_{A}+s^{\prime} H_{B}+F
$$

where

$$
t^{\prime}=t \alpha_{0}+s \beta_{0}, \quad s^{\prime}=-t \beta_{0}+s \alpha_{0},
$$

$\alpha_{0}, \beta_{0}$ being the values of $\alpha, \beta$ at $\gamma^{0}$ and $F$ is a linear combination of $H_{\alpha}$ and $H_{\beta}$ with coefficients having $A$ or $B$ as factors, plus a linear combination of $H_{A}$ and $H_{B}$ with coefficients vanishing at $\gamma^{0}$. We can now prove by induction on the integer $p \geqslant 1$, that

$$
\left(t H_{\tilde{A}}+s H_{\tilde{B}}\right)^{p}=\left(t^{\prime} H_{A}+s^{\prime} H_{B}\right)^{p}+G
$$

where $G$ is a linear combination of differential operators of the form $H_{C_{1}} H_{C_{2}} \cdots H_{C_{\text {, }}}$, $j=1, \ldots, p$, with $C_{l}$ being $\alpha, \beta, A$ or $B$, and coefficients satisfying the following:

(i) If in a term all the $C_{l}$ are $A$ or $B$, then either $j<p$ or $j=p$ and the coefficient of that term vanishes at $\gamma^{0}$.

(ii) If in a term at least one of the $C_{l}$ is $\alpha$ or $\beta$, then the coefficient of that term contains a factor of the form $H_{E_{1}} \cdots H_{E_{h}} A$ or $H_{E_{1}} \cdots H_{E_{h}} B$ with $E_{l}$ being $A$ or $B$ and $h<p$.

We also have

$$
H_{\tilde{A}} \tilde{B}=\left(\alpha^{2}+\beta^{2}\right) H_{A} B+Q
$$

where $Q$ is a sum of terms having $A$ or $B$ as factors. 
Applying now (3.4) with $p=k-1$ to (3.5) and using the definition (1.4) of $k$, and the forms of $G$ and $Q$ we obtain the formula

$$
\left.\left(t H_{\tilde{A}}+s H_{\tilde{B}}\right)^{k-1} H_{\tilde{A}} \tilde{B}\right|_{\gamma^{0}}=\left.\left(\alpha_{0}^{2}+\beta_{0}^{2}\right)\left(t^{\prime} H_{A}+s^{\prime} H_{B}\right)^{k-1} H_{A} B\right|_{\gamma^{0}} .
$$

On the other hand, from (3.3) we have

$$
v_{t t}+v_{s s}=\left(\alpha_{0}^{2}+\beta_{0}^{2}\right)\left(v_{t^{\prime} t^{\prime}}+v_{s^{\prime} s^{\prime}}\right) .
$$

Thus, equation (2.2) for $\tilde{p}_{m}$ is

$$
v_{t^{\prime} t^{\prime}}+v_{s^{\prime} s^{\prime}}=\left.\left(t^{\prime} H_{A}+s^{\prime} H_{B}\right)^{k-1} H_{A} B\right|_{\gamma^{0}}
$$

which is equation (2.2) for $p_{m}$, with $t, s$ replaced by $t^{\prime}, s^{\prime}$. The proof of the lemma is now complete.

4. Reduction of the principal symbol. The factorization of the principal symbol was carried out in [4]. We repeat here the result in a slightly simplified notation and with a more convenient choice of signs.

Lemma 4.1. Suppose assumptions (I) and (II) of Theorem 2.1 hold. Then in a neighborhood $U$ of any point of $M$ we can make an analytic change of variables with the new variables centered at that point and denoted by

$$
(x, t, s, y)=\left(x, t, s, y_{1}, \ldots, y_{l}\right), \quad 3+l=n,
$$

such that in $U, S$ is given by $x=0$ and $M$ by

$$
M: x=t=s=0
$$

and in $T^{*} U, \Sigma$ is given by

$$
\Sigma: x=t=s=0, \quad \tau=\sigma=0, \quad \eta=0,
$$

where $\xi, \tau, \sigma, \eta$ are the dual variables of $x, t, s, y$. Moreover in a neighborhood $V$ of the point $\gamma^{0} \in \Sigma$ given by

$$
\gamma^{0}: x=t=s=0, \quad y=0, \quad \xi=1, \quad \tau=\sigma=0, \quad \eta=0,
$$

the principal symbol $p_{m}=p_{m}(x, t, s, y, \xi, \tau, \sigma, \eta)$ can be written in the form

$$
p_{m}=q[\tau-a+i(\sigma-b+g)]
$$

where $q=q(x, t, s, y, \xi, \tau, \sigma, \eta), a=a(x, t, s, y, \xi, \sigma, \eta), b=b(x, s, y, \xi, \sigma, \eta)$, and $g=g(x, t, s, y, \xi, \sigma, \eta)$ are analytic functions of their arguments, homogeneous with respect to $\xi, \tau, \sigma, \eta$ of degree $m-1$ for $q$ and 1 for $a, b$ and $g ; q$ is complex valued and nonvanishing while $a, b$ and $g$ are real valued and satisfy

$$
\begin{gathered}
a(x, t, s, y, \xi, 0,0)=0, \quad \operatorname{grad} a(x, t, s, y, \xi, 0,0)=0, \\
b(x, s, y, \xi, 0,0)=0, \quad \operatorname{grad} b(x, s, y, \xi, 0,0)=0, \\
\left.g(x, t, s, y, \xi, \sigma, \eta)\right|_{t=0}=0 .
\end{gathered}
$$

Since the assumptions of Theorem 2.1 are invariant under multiplication by a nonvanishing factor, these assumptions can be transferred to $q^{-1} p_{m}$. This allows us to assume from now on that $q \equiv 1$ in (4.4). 
LEMMA 4.2. Suppose that in addition to assumptions (I) and (II) we also have, for all $\gamma \in \Sigma$,

$$
k(\gamma, A, B) \geqslant k_{0}
$$

where $k_{0}$ is a fixed integer $\geqslant 1$. Then

$$
\left.\partial_{t}^{\alpha} \partial_{s}^{\beta} g\right|_{\Sigma}=0 \quad \text { if } 0 \leqslant \alpha+\beta \leqslant k_{0}-1
$$

and

$$
\left.\left(\bar{t} H_{A}+\bar{s} H_{B}\right)^{k_{0}-1} H_{A} B\right|_{\Sigma}=\left.\left(\bar{t} \partial_{t}+\bar{s} \partial_{s}\right)^{k_{0}-1} \partial_{t} g\right|_{\Sigma}
$$

for all $(\bar{t}, \bar{s}) \in \mathbf{R}^{2}$.

Proof. From (4.4) with $q \equiv 1$ we have $A=\tau-a, B=\sigma-b+g$. Hence

$$
H_{A} B=\partial_{t} g-H_{a} B
$$

and

$$
\bar{t} H_{A}+\bar{s} H_{B}=\partial_{r}+H_{e}
$$

where

$$
\partial_{r}=\bar{t} \partial_{t}+\bar{s} \partial_{s}
$$

and

$$
e=\bar{s} g-\bar{t} a-\bar{s} b .
$$

We will prove Lemma 4.2 by induction on $k_{0}$. First, for $k_{0}=1,(4.9)$ follows from (4.7) and (4.10) from (4.11) and (4.5). Assuming now that the lemma is valid for $k_{0}$ we will prove it for $k_{0}+1$. We must show that

$$
k(\gamma, A, B) \geqslant k_{0}+1, \quad \gamma \in \Sigma,
$$

implies that

$$
\left.\partial_{t}^{\alpha} \partial_{s}^{\beta} g\right|_{\Sigma}=0 \text { if } 0 \leqslant \alpha+\beta \leqslant k_{0}
$$

and

$$
\left.\left(\bar{t} H_{A}+\bar{s} H_{B}\right)^{k_{0}} H_{A} B\right|_{\Sigma}=\left.\left(\bar{t} \partial_{t}+\bar{s} \partial_{s}\right)^{k_{0}} \partial_{t} g\right|_{\Sigma} .
$$

From the induction hypothesis and since $k_{0}+1>k_{0}$ we know that (4.9) and (4.10) hold. Thus in order to prove (4.16) it is only necessary to show it for $\alpha+\beta=k_{0}$. But this follows from

$$
\left.\left(\bar{t} \partial_{t}+\bar{s} \partial_{s}\right)^{k_{0}-1} \partial_{t} g\right|_{\Sigma}=0 ; \quad(\bar{t}, \bar{s}) \in \mathbf{R}^{2},
$$

which results from (4.10) using (4.15) and the definition (1.4) of $k$. (Note that from (4.7) we have $\left.\partial_{s}^{j} g\right|_{\Sigma}=0$ for all $j$.)

It remains to prove (4.17). From (4.12) we have $\left(\bar{t} H_{A}+\bar{s} H_{B}\right)^{k_{0}}=\left(\partial_{r}+H_{e}\right)^{k_{0}}$. Expanding the right side and using repeatedly the formula $\partial_{r} H_{e}=H_{\partial_{r} e}+H_{e} \partial_{r}$ we find that

$$
\begin{aligned}
& \left(\bar{t} H_{A}+\bar{s} H_{B}\right)^{k_{0}}=\partial_{r}^{k_{0}}+\text { a linear combination with integer } \\
& \text { coefficients of terms of the form } H_{\partial_{r}^{j l e}} H_{\partial_{r}^{\prime} 2 e} \cdots H_{\partial_{r}^{j} p e} \partial_{r}^{j_{0}}
\end{aligned}
$$


where $j_{0}+j_{1}+\cdots+j_{p}+p=k_{0} ; j_{i} \geqslant 0, i=0,1, \ldots, p ; p \geqslant 1$. Now, for any $j$, we have from (4.13) and (4.14),

$$
H_{\partial_{r}^{j} e}=F_{\xi} \partial_{x}+F_{\sigma} \partial_{s}+F_{\eta} \partial_{y}-F_{x} \partial_{\xi}-F_{t} \partial_{\tau}-F_{s} \partial_{\sigma}-F_{y} \partial_{\eta}
$$

where $F=\left(\bar{t} \partial_{t}+\bar{s} \partial_{s}\right)^{j}(\bar{s} g-\bar{t} a-\bar{s} b)$. Since we always have $j_{1} \leqslant k_{0}-p \leqslant k_{0}-1$, we conclude, using (4.5), (4.6), (4.16) and the fact that $y$ and $\xi$ are free on $\Sigma$, that

$$
\left.H_{\partial_{r}^{\prime \prime l} e}\right|_{\Sigma}=\left.F_{\sigma}\right|_{\Sigma} \partial_{s}+\left.F_{\eta}\right|_{\Sigma} \partial_{y}-\left.F_{x}\right|_{\Sigma} \partial_{\xi}=P_{1}\left(\partial_{s}, \partial_{y}, \partial_{\xi}\right)
$$

where $P_{i}$ denotes an $i$ th order linear operator in the indicated derivatives. Next, since $j_{1}+j_{2} \leqslant k_{0}-p \leqslant k_{0}-2$, we always have $j_{2} \leqslant k_{0}-2$, and using (4.20), (4.21), (4.5), (4.6) and (4.16) we find that

$$
\left.H_{\partial_{r}^{\prime} 1 e} H_{\partial_{r}^{\prime}{ }^{\prime} e}\right|_{\Sigma}=P_{2}\left(\partial_{s}, \partial_{y}, \partial_{\xi}\right)
$$

Proceeding in this fashion we conclude that

$$
\left.H_{\partial_{r}^{j} l_{e}} H_{\partial_{r}^{j_{2}} e} \cdots H_{\partial_{r}^{j} p e}\right|_{\Sigma}=P_{p}\left(\partial_{s}, \partial_{y}, \partial_{\xi}\right) \text {. }
$$

Of course some or all of the coefficients in these operators may be zero. In fact, when $j_{1}=0$ all of the coefficients vanish since $\left.H_{e}\right|_{\Sigma}=0$ as follows from (4.21), (4.5), (4.6) and (4.7). Consequently, dropping in (4.19) the terms with $j_{1}=0$ and using the fact that in the remaining terms we must have $j_{0}+p \leqslant k_{0}-1$, we conclude that

$$
\left(\bar{t} H_{A}+\bar{s} H_{B}\right)^{k_{0}}=\partial_{r}^{k_{0}}+P_{k_{0}-1}\left(\partial_{t}, \partial_{s}, \partial_{y}, \partial_{\xi}\right) .
$$

Applying now (4.23) to (4.11) and using (4.16) and (4.5), we obtain (4.17). The proof of Lemma 4.2 is complete.

We are now ready to express conditions (III) and (IV) of our theorem in terms of the function $g$.

LEMMA 4.3. Suppose that all the assumptions of Theorem 2.1 are satisfied. Then

$$
g(0, t, s, y ; 1,0,0)=g_{k}(t, s ; y)+(\text { terms of degree } \geqslant k+1 \text { in } t, s)
$$

where $g_{k}$ is a homogeneous polynomial of degree $k$ in $t, s$ with coefficients analytic in $y$ and $g_{k}(0, s ; y)=0$. Moreover for each $y$ near 0 , the equation

$$
v_{t t}+v_{s s}=\partial_{t} g_{k}(t, s ; y)
$$

has a homogeneous solution $v(t, s ; y)$ having a strong extremum when $(t, s)=(0,0)$.

Proof. (4.24) follows immediately from assertion (4.9) of Lemma 4.2 with $k=k_{0}$. Since $\partial_{t} g_{k}(t, s ; y)$ consists of the terms of degree $k-1$ in the $(t, s)$-expansion of $\partial_{t} g(0, t, s, y ; 1,0,0)$, we have from Taylor's formula,

$$
\partial_{t} g_{k}(\bar{t}, \bar{s} ; y)=\left.\frac{1}{(k-1) !}\left(\bar{t} \partial_{t}+\bar{s} \partial_{s}\right)^{k-1} \partial_{t} g\right|_{\Sigma, \xi=1} .
$$

Combining (4.26) and assertion (4.10) of Lemma 4.2 with $k=k_{0}$, we find that

$$
\left.\frac{1}{(k-1) !}\left(t H_{A}+s H_{B}\right)^{k-1} H_{A} B\right|_{\Sigma, \xi=1}=\partial_{t} g_{k}(t, s ; y) \text {. }
$$


The last assertion of Lemma 4.3 follows now from hypothesis (IV) of Theorem 2.1, and the fact that this hypothesis is invariant under multiplication of the right side of equation (2.2) by a nonvanishing factor.

REMARK. Since $g$ is homogeneous of degree 1 with respect to $\xi, \sigma, \eta$, possibly after replacing $\xi=1$ by $\xi=-1$ in the definition (4.3) of the point $\gamma^{0}$, we may assume without loss of generality that equation (4.25) has a homogeneous solution with a strong maximum when $(t, s)=(0,0)$.

\section{Construction of the phase function.}

LEMmA 5.1. Suppose that the functions $a, b$ and $g$ have the properties described in Lemmas 4.1 and 4.3. Suppose also that for y near 0, equation (4.25) has a homogeneous solution with a strong maximum when $(t, s)=(0,0)$. Then there are real-valued analytic functions $C_{1}=C_{1}(y)$ and $C_{2}=C_{2}(y)$ defined near $y=0$ such that the solution of the Cauchy problem

$$
\begin{gathered}
\phi_{t}-a\left(x, t, s, y, \phi_{x}, \phi_{s}, \phi_{y}\right)+i \phi_{s} \\
-i b\left(x, s, y, \phi_{x}, \phi_{s}, \phi_{y}\right)+i g\left(x, t, s, y, \phi_{x}, \phi_{s}, \phi_{y}\right)=0, \\
\phi(x, 0, s, y)=x+i x^{2}+\left[C_{1}(y)+i C_{2}(y)\right] s^{k+1}
\end{gathered}
$$

has the property

$$
\begin{aligned}
& \operatorname{Re} \phi(x, t, s, y)=0 \quad \text { implies } \\
& \operatorname{Im} \phi(x, t, s, y) \geqslant K\left[x^{2}+\left(t^{2}+s^{2}\right)^{(k+1) / 2}\right]
\end{aligned}
$$

in some neighborhood of the origin of $\mathbf{R}^{n}$, for some positive constant $K$.

Proof. Expanding the solution $\phi$ of (5.1), (5.2) in powers of $x$ we find that

$$
\phi(x, t, s, y)=x+i x^{2}+\phi_{0}(t, s, y)+\phi_{1}(t, s, y) x+O\left(x^{2}|t|\right)
$$

where

$$
\phi_{0}(0, s, y)=\left[C_{1}(y)+i C_{2}(y)\right] s^{k+1}, \quad \phi_{1}(0, s, y)=0 .
$$

Substituting (5.4) into (5.1) and setting $x=0$ we obtain

$$
\begin{aligned}
\partial_{t} \phi_{0}-a\left(0, t, s, y, 1+\phi_{1}, \partial_{s} \phi_{0}, \partial_{y} \phi_{0}\right)+i \partial_{s} \phi_{0} \\
-i b\left(0, t, s, y, 1+\phi_{1}, \partial_{s} \phi_{0}, \partial_{y} \phi_{0}\right) \\
\quad+i g\left(0, t, s, y, 1+\phi_{1}, \partial_{s} \phi_{0}, \partial_{y} \phi_{0}\right)=0 .
\end{aligned}
$$

Expanding the functions $a, b$ and $g$ about $\sigma=0, \eta=0$ and using (4.5)-(4.7) and the homogeneity of $g$, we can write equation (5.6) in the form

$$
\begin{aligned}
\partial_{t} \phi_{0} & +i \partial_{s} \phi_{0}+i g(0, t, s, y, 1,0,0)\left(1+\phi_{1}\right) \\
& +\left(\text { terms with second degree factors in } \partial_{s} \phi_{0}, \partial_{y} \phi_{0}\right) \\
& +\left(\text { terms with factors } t \partial_{s} \phi_{0} \text { or } t \partial_{y} \phi_{0}\right)=0 .
\end{aligned}
$$

Now substituting in (5.7) the expansion of $\phi_{0}$ in $t$ and $s$ and the expansion (4.24) for $g$, and then successively setting to zero the coefficients of the terms of degree 
$0,1, \ldots, k-1$, we easily conclude that the expansion of $\phi_{0}$ must begin with terms of degree $k+1$. Thus

$$
\begin{aligned}
\phi_{0}(t, s ; y)= & u(t, s ; y)+i v(t, s ; y) \\
& +(\text { terms of degree } \geqslant k+2 \text { in } t \text { and } s)
\end{aligned}
$$

where $u$ and $v$ are real homogeneous polynomials of degree $k+1$ in $t$ and $s$ with coefficients analytic functions of $y$. Substituting (5.8) and (4.24) in (5.7) and retaining only the terms of degree $k$ we obtain

$$
\partial_{t}(u+i v)+i \partial_{s}(u+i v)+i g_{k}=0 .
$$

Also substituting (5.8) in (5.5) we find that

$$
\left.(u+i v)\right|_{t=0}=\left(C_{1}+i C_{2}\right) s^{k+1} .
$$

Separating the real and imaginary parts of (5.9) and (5.10) we have

$$
\begin{aligned}
u_{t}-v_{s} & =0, \\
v_{t}+u_{s} & =-g_{k}, \\
\left.u\right|_{t=0} & =C_{1} s^{k+1}, \\
\left.v\right|_{t=0} & =C_{2} s^{k+1} .
\end{aligned}
$$

Eliminating $u$ between (5.11) and (5.12) we find that $v$ must satisfy the equation

$$
v_{t t}+v_{s s}=-\partial_{t} g_{k} \text {. }
$$

We now use our assumption concerning equation (4.25) which implies that equation (5.15) has a homogeneous solution with a strong minimum when $(t, s)=(0,0)$. Let this solution be given by

$$
v(t, s ; y)=\sum_{\substack{j \geq 0, l \geq 0 \\ j+l=k+1}} c_{j l}(y) t^{j} s^{l} .
$$

In order to satisfy (5.14) we must take

$$
C_{2}(y)=c_{0, k+1}(y) \text {. }
$$

Substituting (5.16) in (5.11), integrating with respect to $t$ and using (5.13) we find

$$
u(t, s ; y)=C_{1}(y) s^{k+1}+\sum_{\substack{j \geqslant 0, l \geqslant 1 \\ j+l=k+1}} \frac{l}{j+1} c_{j l} t^{j+1} s^{l-1} .
$$

We will now determine $C_{1}$ by satisfying (5.12). First we find $g_{k}$ by substituting (5.16) in (5.15), integrating with respect to $t$ and using $\left.g_{k}\right|_{t=0}=0$. Then we substitute in (5.12) the resulting expression for $g_{k}$ and the expressions for $v_{t}$ and $u_{s}$ from (5.16) and (5.18). This yields

$$
C_{1}(y)=-\frac{1}{k+1} c_{1, k}(y) .
$$

From the uniqueness of solution of the Cauchy problem (5.11)-(5.14) we conclude that if we choose $C_{1}$ and $C_{2}$ according to formulas (5.17) and (5.19), then $u$ and $v$ must be given by (5.16) and (5.18). Moreover, from the minimum property of $v$ and 
(5.8), it follows that there is a constant $K_{1}>0$ such that

$$
\operatorname{Im} \phi_{0}(t, s ; y) \geqslant K_{1}\left(t^{2}+s^{2}\right)^{(k+1) / 2}
$$

near $(t, s, y)=(0,0,0)$.

We are now ready to complete the proof of Lemma 5.1. From (5.4) we have

$$
\begin{aligned}
& \operatorname{Re} \phi=x\left(1+\operatorname{Re} \phi_{1}\right)+\operatorname{Re} \phi_{0}+O\left(x^{2}|t|\right), \\
& \operatorname{Im} \phi=x^{2}+\operatorname{Im} \phi_{0}+\left(\operatorname{Im} \phi_{1}\right) x+O\left(x^{2}|t|\right) .
\end{aligned}
$$

Thus, when $\operatorname{Re} \phi=0$, it follows from (5.21) and (5.8) that

$$
x=(\text { terms of degree } \geqslant k+1 \text { in } t, s)+O\left(x^{2}|t|\right) .
$$

Substituting (5.20) and (5.23) in (5.22) and remembering that $\phi_{1}(0, s, y)=0$, the conclusion of the lemma follows immediately.

\section{REFERENCES}

1. M. S. Baouendi, F. Treves and E. C. Zachmanoglou, Flat solutions and singular solutions of homogeneous linear partial differential equations with analytic coefficients, Duke Math. J. 46 (1979), 409-440.

2. L. Hörmander, Subelliptic operators, Seminar on Singularities of Solutions of Linear Partial Differential Equations (L. Hörmander, editor), Ann. of Math. Studies, No. 91, Princeton Univ. Press, Princeton, N. J., 1979.

3. E. C. Zachmanoglou, Manifolds with arbitrary submanifolds of nonanalyticity of solutions of linear $P D E$ 's, Comm. Partial Differential Equations, 5 (1980), 225-243.

4. Manifolds of nonanalyticity of solutions of certain linear PDE's, Trans. Amer. Math. Soc. 266 (1981), 573-582.

Department of Mathematics, Purdue University, West Lafayette, Indiana 47907 